\title{
REVIEW
}

\section{Amino acids, taste circuits, and feeding behavior in Drosophila: towards understanding the psychology of feeding in flies and man}

\author{
Christoph Melcher, Ruediger Bader and Michael J Pankratz \\ Institute for Genetics, Forschungszentrum Karlsruhe, Karlsruhe, Germany \\ (Requests for offprints should be addressed to M J Pankratz; Email: michael.pankratz@itg.fzk.de) \\ (C Melcher is now at Department of Neurobiology, Harvard Medical School, Boston, Massachusetts, USA)
}

\begin{abstract}
Feeding can be regulated by a variety of external sensory stimuli such as olfaction and gustation, as well as by systemic internal signals of feeding status and metabolic needs. Faced with a major health epidemic in eating-related conditions, such as obesity and diabetes, there is an ever increasing need to dissect and understand the complex regulatory network underlying the multiple aspects of feeding behavior. In this minireview, we highlight the use of Drosophila in studying the neural circuits that control the feeding behavior in response to
\end{abstract}

external and internal signals. In particular, we outline the work on the neuroanatomical and functional characterization of the newly identified hugin neuronal circuit. We focus on the pivotal role of the central nervous system in integrating external and internal feeding-relevant information, thus enabling the organism to make one of the most basic decisions - to eat or not to eat.

Journal of Endocrinology (2007) 192, 467-472

\section{Introduction}

Feeding is a fundamental activity of all animals. The precise nature of feeding, such as meal size and frequency, foraging strategy, and food preference, can vary enormously across the animal kingdom, but the basic goal is the same - food intake must satisfy the growth, survival, and reproductive requirements of the organism. This has resulted in animals having evolved specialized feeding habits based on their specific internal metabolic needs and external sensory signals. Many are obvious behaviors, such as looking for food when hungry. Others involve more subtle or difficult choices, such as deciding between similar foods or between feeding and being fed upon by potential predators. An interesting biological problem is how the central nervous system (CNS) processes relevant information to generate appropriate behavioral responses.

This issue is not only of basic biological interest, but also has major medical and agricultural implications. Changing food choices in modern developed countries, together with changes in lifestyle, have helped create major epidemics in conditions such as diabetes and obesity. The nervous system that has evolved to survive under conditions of food scarcity must now also deal with food and caloric excess. From an agricultural side, insects cause immense damage to crops. This problem is especially acute, precisely in regions where malnutrition and starvation pose the greatest threat. And in contrast to those of developed countries, many of the major health problems of underdeveloped countries stem from insect-transmitted diseases, such as malaria.

In this review, we highlight the use of Drosophila in studying neural circuits that control food intake. In particular, we outline the work in our laboratory that emphasizes the use of Drosophila molecular genetics in bridging studies of traditional physiological insect models with those emerging in vertebrates, focusing on the newly identified hugin neural circuit.

\section{Drosophila larval mutants defective in feeding behavior}

Drosophila offers a three-for-one deal as a model for studying the feeding behavior. During the larval stage, Drosophila feed almost continuously, accompanied by a huge increase in mass. In this stage, there is little sex-dependent behavior. In the adult stage, the flies feed intermittently and do not grow in mass. Furthermore, sex differences in their feeding behavior become apparent, as males and females have different metabolic needs. Females have greater biosynthetic needs, since they produce large numbers of eggs over many weeks; males do not have such metabolic demands and their feeding behavior is altered accordingly (Carvalho et al. 2006). 
To gain an entry point into the genes that could be involved in the feeding behavior, we carried out a genetic screen for larval mutants that could not feed. The screen was based on the fact that larvae are translucent; therefore, feeding them with dyed yeast allowed easy visualization of food intake. One of the mutants identified was pumpless ( $p p l$, where the food is not swallowed from the pharynx into the esophagus (Zinke et al. 1999). These mutants have neither morphological defects in the feeding apparatus nor general locomotor defects, and they die as small larvae. They also display another altered feeding behavior, in that they move away from the food source. When wild-type larvae are placed on an agar surface with yeast paste in the middle, they spend most of their time buried in the yeast. By contrast, $p p l$ larvae move about actively outside the yeast paste. In addition, $p p l$ larvae do not show normal physiological starvation responses such as, for example, upregulation of lipase-encoding genes (Zinke et al. 2002). These observations gave rise to the idea that $p p l$ animals feel 'full' and therefore stop feeding prematurely. The $p p l$ gene encodes a subunit of the glycine cleavage system, which catabolizes the amino acid glycine; $p p l$ is expressed specifically in the fat body, an organ analogous to the vertebrate liver and adipocytes. Feeding wild-type animals with high levels of glycine and other amino acids also causes cessation of feeding and growth, and we proposed that some amino-acid-dependent signal from the fat body acts on the CNS to bring about the cessation of feeding (Zinke et al. 1999).

Subsequently, several other genetic manipulations were reported to trigger such wandering-like behavior. For example, overactivation of insulin receptor/PI3K signaling as well as overexpression of $\mathrm{dFOXO}$, a direct mediator of insulin signaling, suppresses the growth and brings about larval wandering (Britton et al. 2002, Junger et al. 2003, Kramer et al. 2003). The expression of $p p l$ is altered by nutrient conditions, suggesting it could also be a target of insulin signaling, but this remains to be determined (Zinke et al. 1999).

Intriguingly, a human condition is known, which derives from an inborn error of the glycine cleavage system, termed nonketotic hyperglycinemia. Some clinical features of nonketotic hyperglycinemia are described as follows (taken from (Nyhan 1989)): 'Most affected infants appear normal at birth and for a short interval, seldom longer than $48 \mathrm{~h} .$. . After this interval, which may or may not await the initiation of protein-containing feedings, the infant develops lethargy and/ or convulsions... With increasing lethargy there is anorexia leading to failure to feed'.

Patients with nonketotic hyperglycinemia have elevated glycine levels in blood and urine. The level of free glycine in the brain is also elevated; the ratio of cerebrospinal fluid concentration to that of plasma is tenfold higher than normal (Nyhan 1989). As glycine is an inhibitory neurotransmitter, many of the neurological consequences, including feeding defects, could be due to the direct effects of high glycine on the nervous system. In Drosophila, defects in glycine cleavage may lead to accumulation of glycine in the hemolymph, which could then act on the CNS to effect stoppage of feeding. We have recently obtained evidence that free glycine level in ppl mutants is indeed increased (M J Pankratz, unpublished data). Furthermore, recent genetic manipulations in mice showed that altering amino-aciddependent signaling pathways in the brain, such as target of rapamycin and GCN2-kinase pathways, also result in feeding behavior defects (Hao et al. 2005, Maurin et al. 2005, Cota et al. 2006).

In addition to acting as internal metabolic signals, amino acids may also influence the feeding behavior through altering external sensory perception. One of the most commonly used taste enhancers is the amino acid, glutamate, which generally acts as an excitatory neurotransmitter. The role of monosodium glutamate as a flavor enhancer is well known. Growing up in a Korean household (one of the authors), a bottle of 'ajinomoto', a brand name for monosodium glutamate, was a standard presence on the dining table. As amino acids are the building blocks for driving cellular and organismal growth, it is not surprising that they should have such profound effects on taste and feeding response in the CNS.

\section{Neuromedins and hugin}

We recently showed that another larval feeding mutant identified in our screen was due to a mutation in the klumpfuss $(k l u)$ locus (Melcher \& Pankratz 2005). The klu mutants show a similar feeding phenotype as $p p l$, including the wandering-like phenotype. The gene encodes a zinc finger transcription factor and is expressed throughout the nervous system. Microarray analysis focusing on neuropeptide gene regulation indicated that hugin was upregulated in klu mutants; hugin is expressed exclusively in the brain (Meng et al. 2002). It is upregulated in ppl larvae as well, and its expression in wild type is downregulated under amino-acid-deprived conditions, suggesting that hugin may act in the brain to modulate the feeding behavior in response to amino acid signals (Melcher \& Pankratz 2005).

The hugin gene encodes a prepropeptide that can be cleaved into several neuropeptides, one named PK-2 and another hugin- $\gamma$ (Meng et al. 2002). Recently, we suggested that hugin is a homolog of human neuromedin $\mathrm{U}(\mathrm{NmU}$; Melcher et al. 2006). Sequence analysis based on one of the hugin cleavage products, PK-2, revealed structural homologies between mammalian sequences and those from other insects (Fig. 1). Mammalian $\mathrm{NmU}$ is closely related to another neuropeptide encoding gene, neuromedin S (NmS; Mori et al. 2005), with the last seven amino acids being identical in all cases. For the moth Helicoverpa zea, the pheromone biosynthesis-activating neuropeptide (PBAN)-encoding gene neuropeptide (PGN-8) derives from the same prepropeptide that generates PBAN; (Choi et al. 2003, Hull et al. 2004). From additional sequence analysis and functional studies on bioactive properties of these neuropeptides, it seems that there is considerable variation in the length of the $\mathrm{N}$-terminal region, and that the most conserved and potent bioactive region lies in the last 7-9 amino acids. 


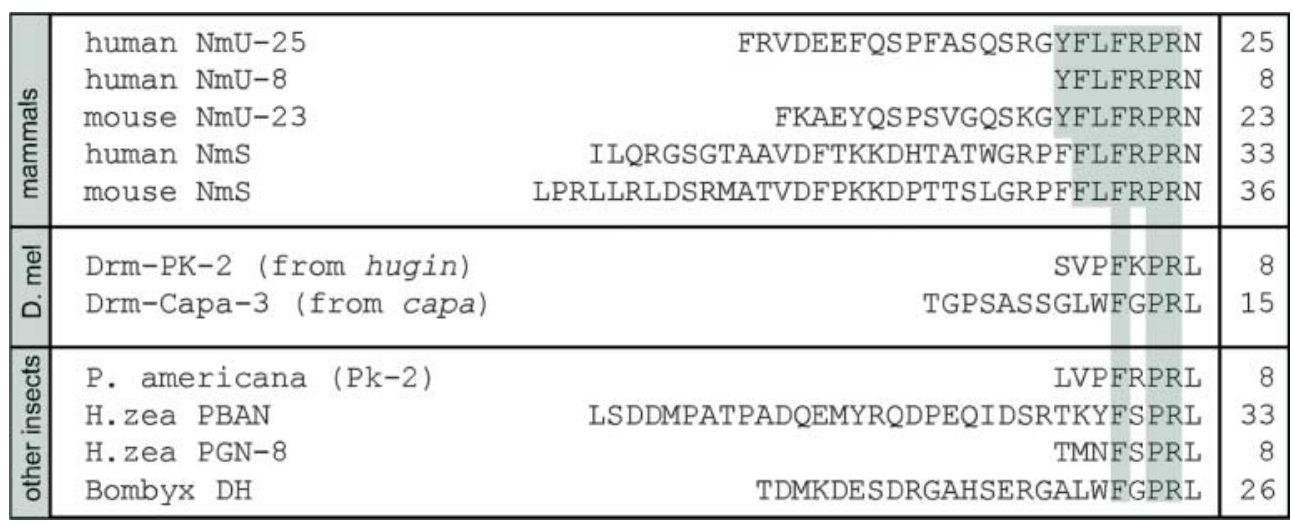

Figure 1 Amino acid comparisons of neuromedin U-related neuropeptides in vertebrates and insects. Species, protein name, amino acid sequence of bioactive peptides, and peptide size (in numbers of amino acids) are shown. The conserved amino acids are highlighted by gray shading. The human NmU-8 protein is entirely contained in the larger NmU-25.

Similarities between these neuropeptides extend well in to the functional levels, including brain-specific expression domains as well as feeding-related mis- and overexpression phenotypes (Melcher et al. 2006).

The G-protein-coupled receptors (GPCRs) for these neuropeptides are also highly homologous. Mammalian $\mathrm{NmU}$ and $\mathrm{NmS}$ can activate the same set of receptors, and many of the $\mathrm{NmU}$ family of neuropeptides from one species can activate the receptors from another (Howard et al. 2000, Park et al. 2002, Mori et al. 2005). Two GPCRs in the Drosophila genome, CG8795 and CG8784, have been shown to be activated by hugin cleavage products (Park et al. 2002, Rosenkilde et al. 2003). The two receptors share structural similarities and are clustered on the same chromosome (Hewes \& Taghert 2001). Remarkably, $\mathrm{NmU}$ and the $\mathrm{NmU}$ receptor, as well as the $\mathrm{NmS}$, have been shown to regulate the feeding behavior in mammals (Howard et al. 2000, Ivanov et al. 2002, Hanada et al. 2004, Ida et al. 2005), further supporting our view of the Drosophila hugin system and mammalian neuromedin systems being homologous (Melcher et al. 2006).

\section{To eat or not to eat: feeding initiation and tasteful decision making}

Using an artificial hugin promoter to drive expression of various marker genes, we showed that hugin neurons send projections to higher brain centers, neuroendocrine organs, ventral nerve cord, and the pharynx. These results supported the view, at an anatomical level, that hugin neurons play a central role in coordinating feeding, metabolism, and growth (Melcher \& Pankratz 2005). Given this multifariousness of the 'feeding-related' projection targets, a question arose concerning the specificity of hugin neuronal arborizations do all neurons project to all targets, or do subsets of hugin neurons have specific targets? This issue was systematically addressed with clonal analysis of single hugin neurons (Bader et al. 2007). Our analysis demonstrated that each neuron projects to only one of the four major targets (Fig. 2). Moreover, it revealed complexities in neuromorphology that were not apparent before. Most notably, all hugin neurons arborize in the subesophageal ganglion (SOG) just lateral and ventral to the foramen, an opening in the Drosophila brain where the esophagus passes through. It is likely that this anatomical interface between CNS and gastrointestinal tract represents a local site for neuropeptide release. The single cell analysis additionally revealed close intermingling, precisely in the SOG, between some of the hugin neuronal projection termini and those of neurons mediating taste information from external and internal chemosensory organs (Bader et al. 2007). The gustatory receptor neuronal projections arborizing closest to hugin neurons are GR66a positive (Scott et al. 2001). Interestingly, this is a receptor that mediates aversive taste (Thorne et al. 2004, Wang et al. 2004): if GR66aexpressing neurons are rendered nonfunctional by genetic manipulations, flies will feed on aversive substances, such as quinine and caffeine (Thorne et al. 2004), and artificial activation of GR66a taste neurons elicits avoidance behavior to neutral substances (Marella et al. 2006).

The function of the hugin neural circuit was explored in adult flies using tetanus toxin to inhibit neuronal activity in hugin-expressing neurons (Melcher \& Pankratz 2005). The first intriguing result was an earlier initiation of feeding when encountering a previously untested food. In other words, control flies do not feed immediately, but appear to first 'evaluate' the new food through gustatory information, possibly for nutrient content or to avoid toxic substances. Silencing hugin neurons by tetanus toxin eliminated this evaluation phase, resulting in flies that initiated feeding much earlier. This could be phenocopied in control flies by starving them. Challenging control and hugin-tetanus toxin flies with an array of different feeding paradigms further confirmed the emerging picture of a 'to feed or not to feed' decision based on the gustatory cues as well as the feeding status of the fly (Fig. 3). For example, no difference between hugin-tetanus toxin and control flies was seen when they progressed from 

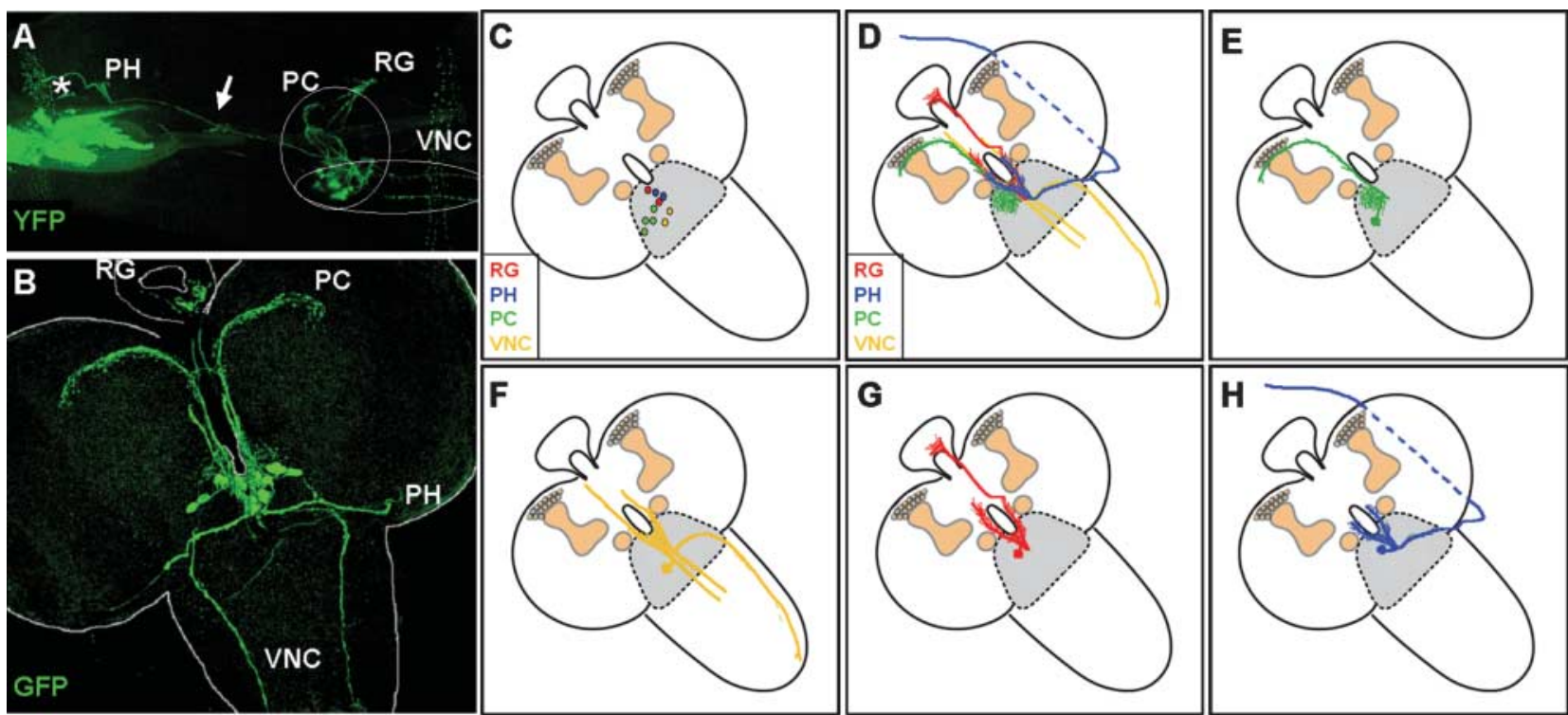

Figure 2 Connectivity of hugin neurous and summary of different hugin neural projection classes. (A) hugin connectivity in a living third instar Drosophila larva using two-photon microscopy. Yellow fluorescent protein (YFP) expressed under a hugin promoter reveals hugin neuronal projections from cell bodies in the SOG to the pharynx ( $\mathrm{PH}$; projection terminal is marked by asterisk and the nerve fiber extending to the head region is marked by arrow), the protocerebrum (PC), the neuroendocrine ring gland (RG), and the ventral nerve cord (VNC). The head is oriented to the left; the strong autofluorescence in the head region stems from the cephalopharyngeal skeleton. Position of the CNS is outlined. (B) Immunohistochemical staining in a third instar larval CNS of GFP expressed under hugin promoter. The four major projection targets are shown. CNS is outlined, anterior points to the upper left. $(\mathrm{C})-(\mathrm{H})$ Schematic summary of hugin neuroanatomy depicting the results of clonal analysis of single hugin neurons (taken from Bader et al. 2007). Relative positions of olfactory centers (comprising mushroom bodies and antennal lobes) are shown in brown and the SOG in gray.

higher quality food to poorer food (e.g. from food containing high yeast to low yeast concentration, or from pure yeast food to yeast food containing aversive substances like quinine). Under these conditions, the flies are already satiated from the higher quality food and do not need to feed on low-quality food immediately. However, a consistent difference was seen when flies were transferred from poorer to better food sources: hugin-tetanus toxin flies initiated feeding earlier than control flies (Melcher \& Pankratz 2005).

One way this decision making based on previous feeding condition or experience could be accomplished is for hugin neuropeptides to be released in the SOG, where they modulate or tune local taste and feeding circuits. Eliminating hugin activity could lower the threshold for initiating feeding, resulting in flies that make the decision to feed earlier. Thus, silencing of sensory receptor neurons responsible for aversive sensation (e.g. GR66a) results in flies feeding on bitter as well as pleasant food. For hugin neurons, which may act as second-order interneurons that process incoming sensory information, blocking their activity would not influence the choice of food, but rather the decision to begin feeding on a certain food.

Acceptance of food based on food quality as well as feeding status of the animal also seems to be regulated by the centrally and peripherally expressed neuropeptide $F$ (npf) and its receptor (npfr; Brown et al. 1999, Shen \& Cai 2001, Wu et al. 2003, 2005a,b). Recent work on the Drosophila npf/npfr system has demonstrated its involvement in suppressing aversion to noxious food in starved larvae (Wu et al. 2005a) as well as in regulating hunger-driven motivational feeding on less accessible solid food (Wu et al. 2005b). Interestingly, there are again striking structural and functional similarities between the Drosophila npf/npfr system and its vertebrate homolog, the neuropeptide $\mathrm{Y} /$ neuropeptide $\mathrm{Y}$ receptor (see Wu et al. 2003 and references therein). It is also noteworthy that npf, which is highly expressed in young third instar larvae attracted to food and whose overexpression delays onset of stop of feeding in old third instar larvae prior to pupariation (Wu et al. 2003), was found to be upregulated in klu mutants that display premature stop of food intake (Melcher \& Pankratz 2005). It remains speculative whether or how, in these mutants, the overactive hugin system overrides npf signaling to cause stop of feeding. One difficulty in integrating the role of the $\mathrm{npf} / \mathrm{npfr}$ system into central regulation of the feeding behavior in Drosophila is its peripheral expression in neuroendocrine cells in the gut (Brown et al. 1999), an issue that has not yet been taken into account at all.

\section{The psychology of feeding behavior in flies and man}

Drosophila can display remarkably human-like behaviors when it comes to feeding. For example, from our above-described studies on adult food intake under different conditions as well as from the work on the role of the npf system on larval 

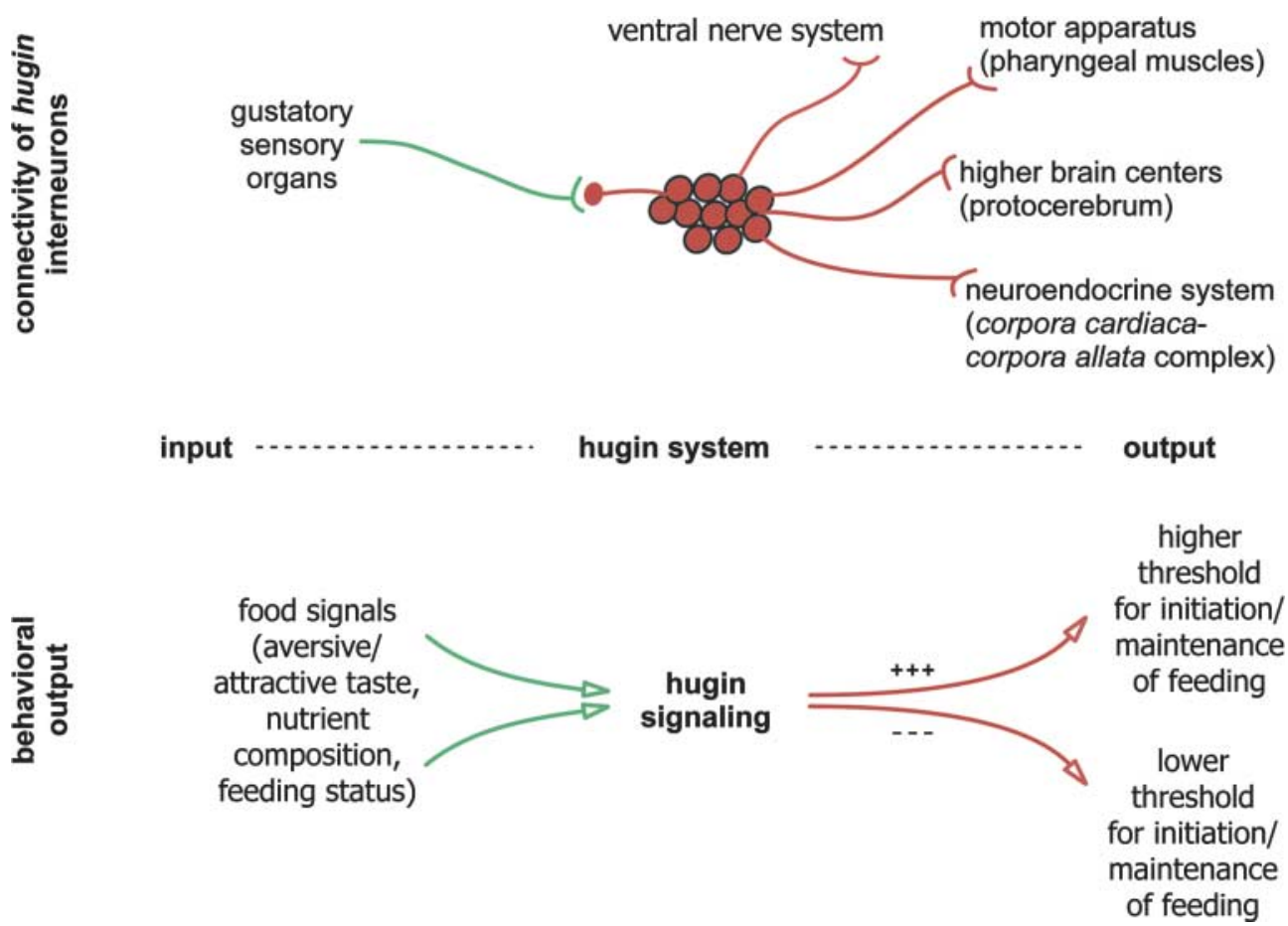

Figure 3 Model of hugin as modulator of feeding behavior. The hugin neurons, which interconnect gustatory sensillae via the SOG to pharyngeal muscles, the protocerebrum, the ring gland, and the ventral nervous system, modulate the feeding behavior in response to food signals such as gustatory information and hunger status. Increased hugin signaling which may lead to an elevated feeding threshold correlates with earlier termination of feeding and food seeking, whereas decreased hugin signaling may lead to a lower feeding threshold and therefore lead to faster initiation of feeding and food seeking. Please see text for details.

feeding behavior, it appears that there are inhibition thresholds for feeding on new or bad-tasting food. If animals are starved, these thresholds will be lowered. If they are starved and given a bad-tasting food, these thresholds will be elevated again (but will still be lower than in well-fed individuals). The length of starvation (i.e. the feeding status) and the quality of food offered are apparently determinants for setting these thresholds. Thus, in times of plenty, one can be picky about what one eats; under hunger conditions, one eats whatever is available, albeit with less gusto. What we have not discussed so far, but which plays a major role in adult feeding behavior, is sex dependence and influence of the reproductive status (see for example, Carvalho et al. 2006). Therefore, in addition to quality of food available and one's hunger status, the decision between feeding and mating is also something that must be considered. It is also noteworthy that in developing Drosophila larvae, the feeding behavior is more robust, suggesting in this case that the metabolic needs have such high priority that more subtle parameters are likely to be overridden and feeding is continuously maintained. Finally, humans often indulge in so-called hedonic feeding, which can manifest, for example, in our continuing to eat a tasty meal although we are 'full' (i.e. all our metabolic needs have been satisfied). It is likely that our ancestors, after hunting down an animal, ate as much as they possibly could in a short period of time, not only because it was difficult to preserve meat, but also due to the uncertainty of the next successful hunt. Thus, although flies may not display hedonic feeding, the study of how flies make food-based decisions may even help us learn about this very human behavior, if we approach the issue from an evolutionary perspective.

\section{Outlook}

A major goal in molecular and neurobiology is to decipher how the brain evaluates multiple, sometimes conflicting, signals to make a decision, and to correlate the resulting behavioral output with specific neural circuits. This requires understanding not only of how a certain gene functions in the context of the whole organism, but also how it functions within identified cells of the brain. This is especially important when working with genes which are expressed not only in parts of the brain but also in peripheral tissues. Which cells are responsible for the behavior that is being studied? Drosophila, with its available genetic tools, is well suited to tackle the problem of understanding central mechanisms that underlie the feeding behavior at the brain circuit level. This, in turn, has implications for two huge, polar opposite issues of our day - the health disorders stemming from nutrient overabundance in developed countries, and crop 
protection and pest control to fight malnutrition and insectborne diseases in developing countries.

\section{Acknowledgements}

We thank all current and previous members of the Pankratz laboratory, especially Mario Gloeck, for lively discussions on fly feeding psychology as well as Rachel Bortnick for critical reading of the manuscript.

\section{Funding}

Work in the Pankratz laboratory was supported by Forschungszentrum Karlsruhe and by grants from Deutsche Forschungsgemeinschaft (DFG). The authors declare that there is no conflict of interest that would prejudice the impartiality of this scientific work.

\section{References}

Bader R, Colomb J, Pankratz B, Schroeck A, Stocker R \& Pankratz MJ 2007 Genetic dissection of a neural circuit anatomy underlying feeding behavior in Drosophila: distinct classes of hugin expressing neurons. Journal of Comparative Neurology (In press).

Britton JS, Lockwood WK, Li L, Cohen SM \& Edgar BA 2002 Drosophila's insulin/PI3-kinase pathway coordinates cellular metabolism with nutritional conditions. Developmental Cell 2 239-249.

Brown MR, Crim JW, Arata RC, Cai HN, Chun C \& Shen P 1999 Identification of a Drosophila brain-gut peptide related to the neuropeptide Y family. Peptides 20 1035-1042.

Carvalho GB, Kapahi P, Anderson DJ \& Benzer S 2006 Allocrine modulation of feeding behavior by the Sex Peptide of Drosophila. Current Biology 16 692-696.

Choi MY, Fuerst EJ, Rafaeli A \& Jurenka R 2003 Identification of a G proteincoupled receptor for pheromone biosynthesis activating neuropeptide from pheromone glands of the moth Helicoverpa zea. PNAS 100 9721-9726.

Cota D, Proulx K, Smith KA, Kozma SC, Thomas G, Woods SC \& Seeley RJ 2006 Hypothalamic mTOR signaling regulates food intake. Science $\mathbf{3 1 2}$ 927-930.

Hanada R, Teranishi H, Pearson JT, Kurokawa M, Hosoda H, Fukushima N, Fukue Y, Serino R, Fujihara H, Ueta Y et al. 2004 Neuromedin U has a novel anorexigenic effect independent of the leptin signaling pathway. Nature Medicine 10 1067-1073.

Hao S, Sharp JW, Ross-Inta CM, McDaniel BJ, Anthony TG, Wek RC, Cavener DR, McGrath BC, Rudell JB, Koehnle TJ et al. 2005 Uncharged tRNA and sensing of amino acid deficiency in mammalian piriform cortex. Science 307 1776-1778.

Hewes RS \& Taghert PH 2001 Neuropeptides and neuropeptide receptors in the Drosophila melanogaster genome. Genome Research 11 1126-1142.

Howard AD, Wang R, Pong SS, Mellin TN, Strack A, Guan XM, Zeng Z, Williams DL Jr, Feighner SD, Nunes CN et al. 2000 Identification of receptors for neuromedin $U$ and its role in feeding. Nature 406 70-74.

Hull JJ, Ohnishi A, Moto K, Kawasaki Y, Kurata R, Suzuki MG \& Matsumoto S 2004 Cloning and characterization of the pheromone biosynthesis activating neuropeptide receptor from the silkmoth, Bombyx mori. Significance of the carboxyl terminus in receptor internalization. Journal of Biological Chemistry 279 51500-51507.

Ida T, Mori K, Miyazato M, Egi Y, Abe S, Nakahara K, Nishihara M, Kangawa K \& Murakami N 2005 Neuromedin s is a novel anorexigenic hormone. Endocrinology 146 4217-4223.
Ivanov TR, Lawrence CB, Stanley PJ \& Luckman SM 2002 Evaluation of neuromedin $\mathrm{U}$ actions in energy homeostasis and pituitary function. Endocrinology 143 3813-3821.

Junger MA, Rintelen F, Stocker H, Wasserman JD, Vegh M, Radimerski T, Greenberg ME \& Hafen E 2003 The Drosophila forkhead transcription factor FOXO mediates the reduction in cell number associated with reduced insulin signaling. Journal of Biology 20.

Kramer JM, Davidge JT, Lockyer JM \& Staveley BE 2003 Expression of Drosophila FOXO regulates growth and can phenocopy starvation. BMC Developmental Biology 35.

Marella S, Fischler W, Kong P, Asgarian S, Rueckert E \& Scott K 2006 Imaging taste responses in the fly brain reveals a functional map of taste category and behavior. Neuron 49 285-295.

Maurin AC, Jousse C, Averous J, Parry L, Bruhat A, Cherasse Y, Zeng H, Zhang Y, Harding HP, Ron D et al. 2005 The GCN2 kinase biases feeding behavior to maintain amino acid homeostasis in omnivores. Cell Metabolism 1 273-277.

Melcher C \& Pankratz MJ 2005 Candidate gustatory interneurons modulating feeding behavior in the Drosophila brain. PLoS Biology 3 e305.

Melcher C, Bader R, Walther S, Simakov O \& Pankratz MJ 2006 Neuromedin $\mathrm{U}$ and its putative Drosophila homolog hugin. PLoS Biology 4 e68.

Meng X, Wahlstrom G, Immonen T, Kolmer M, Tirronen M, Predel R, Kalkkinen N, Heino TI, Sariola H \& Roos C 2002 The Drosophila hugin gene codes for myostimulatory and ecdysis-modifying neuropeptides. Mechanisms of Development 117 5-13.

Mori K, Miyazato M, Ida T, Murakami N, Serino R, Ueta Y, Kojima M \& Kangawa K 2005 Identification of neuromedin $\mathrm{S}$ and its possible role in the mammalian circadian oscillator system. EMBO Journal 24 325-335.

Nyhan W 1989 Nonketotic Hyperglycinemia. edn 6. C Scriver, A Beaudet, W Sly \& D Valle. New York: McGraw-Hill Information Services Company.

Park Y, Kim YJ \& Adams ME 2002 Identification of G protein-coupled receptors for Drosophila PRXamide peptides, CCAP, corazonin, and AKH supports a theory of ligand-receptor coevolution. PNAS 99 11423-11428.

Rosenkilde C, Cazzamali G, Williamson M, Hauser F, Sondergaard L, DeLotto R \& Grimmelikhuijzen CJ 2003 Molecular cloning, functional expression, and gene silencing of two Drosophila receptors for the Drosophila neuropeptide pyrokinin-2. Biochemical and Biophysical Research Communications 309 485-494.

Scott K, Brady R Jr, Cravchik A, Morozov P, Rzhetsky A, Zuker C \& Axel R 2001 A chemosensory gene family encoding candidate gustatory and olfactory receptors in Drosophila. Cell 104 661-673.

Shen P \& Cai HN 2001 Drosophila neuropeptide F mediates integration of chemosensory stimulation and conditioning of the nervous system by food. Journal of Neurobiology 47 16-25.

Thorne N, Chromey C, Bray S \& Amrein H 2004 Taste perception and coding in Drosophila. Current Biology 14 1065-1079.

Wang Z, Singhvi A, Kong P \& Scott K 2004 Taste representations in the Drosophila brain. Cell 117 981-991.

Wu Q, Wen T, Lee G, Park JH, Cai HN \& Shen P 2003 Developmental control of foraging and social behavior by the Drosophila neuropeptide Y-like system. Neuron 39 147-161.

Wu Q, Zhao Z \& Shen P 2005a Regulation of aversion to noxious food by Drosophila neuropeptide Y- and insulin-like systems. Nature Neuroscience $\mathbf{8}$ 1350-1355.

Wu Q, Zhang Y, Xu J \& Shen P 2005b Regulation of hunger-driven behaviors by neural ribosomal S6 kinase in Drosophila. PNAS 102 13289-13294.

Zinke I, Kirchner C, Chao LC, Tetzlaff MT \& Pankratz MJ 1999 Suppression of food intake and growth by amino acids in Drosophila: the role of pumpless, a fat body expressed gene with homology to vertebrate glycine cleavage system. Development 126 5275-5284.

Zinke I, Schutz CS, Katzenberger JD, Bauer M \& Pankratz MJ 2002 Nutrient control of gene expression in Drosophila: microarray analysis of starvation and sugar-dependent response. EMBO Journal 21 6162-6173.

\section{Received in final form 4 January 2007 \\ Accepted 4 January 2007 \\ Made available online as an Accepted Preprint 8 January 2007}

